\title{
Probing the Interplay between AGN Outflows and their Host Galaxies: Optical Integral Field Unit and Radio Imaging
}

\author{
Prajval Shastri ${ }^{1}$ on behalf of the $S 7$ team $^{\dagger}$ \\ ${ }^{1}$ Indian Institute of Astrophysics, Bengaluru 560034 India \\ email: pshastri@iiap.res.in
}

Abstract. We report on the Siding Spring Southern Seyfert Spectroscopic Snap-shot Survey.

\section{The S7 Survey†}

The extreme-ultraviolet photons from accreting supermassive black holes photoionise gas in the nuclear region of the host galaxy, producing high-excitation emission lines. In addition, star-forming regions in the galaxy produce HII regions and associated radio emission. The bipolar synchrotron jets from the active galactic nucleus (AGN) could drive the observed hot gaseous outflows but shock-ionisation could also play a role. Since the nearest AGN hosts can be studied in great detail in the optical waveband from the ground, integral-field spectroscopy can be used to probe abundance gradients across the galaxy as well as the kinematics of the gaseous outflows. Thus the interplay between the AGN outflows and their hosts can be investigated, which is in turn key to understanding the scaling relationships between galaxy bulges and their central black holes. We have initiated such an investigation by studying the morphology, kinematics, excitation and abundance structure of about 120 nearby AGNs using a spectroscopic survey with an Integral Field Unit (WiFeS: Dopita et al.2007, ApSS310, 255) which is mounted on the 2.3m Australia National University telescope at Siding Spring Observatory. The radio structure, which we determine both from archival VLA data and our GMRT observations, probes both star formation and the accreting central black hole. We are thus able to investigate the interaction of the AGN processes with those of the host galaxy. The sample was selected from the Veron-Cetty \& Veron Catalogue of AGN, which is the most comprehensive compilation of known AGN. They were filtered by:

- Declination $<10^{\circ}$ North (accessibility from Siding Spring)

- Galactic lattitude $|\mathrm{bII}|<15^{\circ}$ (for most objects; in order to reduce extinction effects)

- Radio flux density $>20 \mathrm{mJy}$ (to facilate radio imaging follow-up)

- $\mathrm{z}<0.02$ (gives several beams across the galaxy \& the SII line within the WiFeS range)

\section{Summary}

- We have obtained data cubes in the optical wavelength range with a typical seeing of $1^{\prime \prime}-2.5^{\prime \prime}$ for over $60 \%$ of our sample (Dopita et al.2015, ApJSup, 217, 12).

- The spectral resolution of the data, $\mathrm{R}$ is $\sim 3000$ (blue) and $\sim 7000$ (red).

- Our first data release is available at http://miocene.anu.edu.au/priv/S7DR1/

- From our observations so far nearly all the Seyfert galaxies have an extended emissionline region, often within a starformation ring of a few kpc across, which may be interpreted as the Inner Lindblad Resonance.

- When our spatial resolution is sufficient, we see the narrow-line region showing elongated and sometimes biconical morphology, aligned with the inner radio jet.

$\dagger$ The original poster is available at https://www.researchgate.net/publication/283500614 